\title{
Factors associated to serum 25- hydroxyvitamin $D$ levels among older adult populations in urban and suburban communities in Shanghai, China
}

Qun Cheng ${ }^{1,2}$, Yanping Du ${ }^{1,2}$, Wei Hong ${ }^{1,2}$, Wenjing Tang ${ }^{1,2}$, Huilin $\mathrm{Li}^{1,2}$, Minmin Chen ${ }^{1,2}$ and Songbai Zheng ${ }^{2^{*}}$

\begin{abstract}
Background: Vitamin D deficiency is widespread in China, particularly among older adults. Factors associated with suboptimal vitamin D levels are not well defined. The present study was a population-based study that included 10 urban and suburban communities in Shanghai, to evaluate vitamin D status and its correlates among older adults.

Method: This cross-sectional study was based on study data for 3924 healthy men and women aged 65-95 years. Anthropometric and socioeconomic data were collected in June-July 2014. Serum 25(OH)D levels were detected using a chemiluminescence immunoassay. The following socioeconomic data were obtained through selfadministered questionnaires: education level, lifestyle, residency, and dietary habits. A logistic regression model was used to assess associations between anthropometric factors, socioeconomic factors and serum 25(OH)D levels.

Results: Median levels of serum 25(OH)D in men and women were 22.73 and $19.99 \mathrm{ng} / \mathrm{mL}$, respectively. Vitamin D deficiency was common in subjects, even though data collection was conducted during summer. The general prevalence of serum $25(\mathrm{OH}) \mathrm{D}$ levels $<20 \mathrm{ng} / \mathrm{mL}$ were $35.4 \%$ and $50.5 \%$ for men and women respectively. The general prevalence of serum $25(\mathrm{OH})$ D levels $<10 \mathrm{ng} / \mathrm{mL}$ were $2.73 \%$ and $5.9 \%$ for men and women respectively. A multivariable model indicated serum $25(\mathrm{OH}) \mathrm{D}$ levels $\geq 20 \mathrm{ng} / \mathrm{mL}$ were significantly and positively correlated with male sex, calcium or vitamin D supplementation, and residency in suburban communities. The model also indicated that high level of physical activity was protective factors of vitamin D deficiency for men and milk consumption for women, respectively. By contrast, deficient serum 25(OH)D levels were significantly correlated with education level (lower than primary school) or obesity [body mass index (BMI) $\geq 26.06 \mathrm{~kg} / \mathrm{m}^{2}$ ] for men or women, respectively.

Conclusion: This cross-sectional study of older adults in communities in Shanghai demonstrates that key factors positively correlated with serum 25(OH)D levels $\geq 20 \mathrm{ng} / \mathrm{mL}$ include male sex, residency in suburban communities, calcium or vitamin D supplementation, high physical activity and education level (for men), and dairy consumption and maintenance of normal BMI (for women).
\end{abstract}

Keywords: Vitamin D deficiency, Community, Older adults, Sociodemographic factors, Lifestyle, Dietary factors

\footnotetext{
*Correspondence: zhengsongbai@126.com

${ }^{2}$ Research Center on Aging and Medicine, Fudan University, 221 West Yan

An Road, Shanghai 200040, China

Full list of author information is available at the end of the article
} 


\section{Background}

Vitamin D deficiency is coming into a global health problem [1], particularly among young children, pregnant women, and older adults. Vitamin D is crucial for bone and mineral homeostasis, particularly for preventing osteomalacia and secondary hyperparathyroidism in older adults. Vitamin D insufficiency is a potential risk factor of osteoporosis, cardiovascular disease, diabetes, cancer, and autoimmune diseases such as rheumatoid arthritis and multiple sclerosis [2-6]. Considering the significance of vitamin $\mathrm{D}$ for skeletal and extra-skeletal health, optimal vitamin D level should be maintained especially in older adults.

There is growing concern regarding the high prevalence of hypovitaminosis D in China [7]. However, the causes of widespread vitamin D deficiency among older adults in China are not completely clear. As far as we know, few studies have investigated the factors associated with vitamin D levels among the older adult population in China. Information on participating factors of vitamin D status in older adults may facilitate the development of effective interventions. In this study, we selected 3924 subjects (1677 men and 2247 women) aged 65-95 years to assess potential correlations between vitamin D status and sociodemographic and lifestyle factors. Subjects were selected from a community-based osteoporosis prevention study conducted at Huadong Hospital (affiliated with Fudan University), Shanghai, China, during June and July, 2014. Our three aims were as follows: (i) evaluate vitamin D status in a large cohort of adults older than 65 years who were recruited from urban and suburban areas in Shanghai, East China; (ii) investigate the correlations of serum 25-hydroxyvitamin D [25(OH)D] levels with demographic, lifestyle, physical exercise and dietary factors; and (iii) compare potential differences of serum vitamin D levels in residents of urban and suburban areas.

\section{Methods}

\section{Study population}

The present cross-sectional study was based on study data compiled from healthy male and female subjects in 10 communities in and in Shanghai, China (latitude $31^{\circ} \mathrm{N}$; the Shanghai Community Osteoporosis Study), which was conducted from June to July (4 weeks) in 2014. The data consisted mostly of a general health examination and selfreported questionnaires. Health examination data were obtained with standard methods using the same equipment and conducted in a single center.

Subjects recruitment was as previous study [8]. All study subjects were independently ambulant. Subjects who sit in a wheelchair or stayed in bed were excluded from the study. Medical histories and physical examinations confirmed that all participants were healthy old people, without severe diseases interfering with vitamin
D metabolism, such as hyperthyroidism, hyperparathyroidism, hepatic failure, renal failure, or end-stage cancer. Subjects with idiopathic bone disease were also excluded from this study. None of the participants were taking any medications that could affect bone or vitamin D metabolism, such as anti-osteoporotic drugs (e.g., glucocorticoids, heparin, warfarin, thyroxine, sex hormones, bisphosphonates, selective estrogen receptor modulators (SERMs), calcitonin, parathyroid hormone (PTH) analogue, or calcitriol). All subjects were recruited by advertisement, and signed the informed consent before study. The study program was approved by the Huadong Hospital Ethics Committee.

A total of 4864 older adults people from communities in and in Shanghai participated in the health study. We implemented the following exclusion criteria: participants younger than 65 years $(n=167)$, participants without a valid serum $25(\mathrm{OH}) \mathrm{D}$ measurement $(n=102)$, those not finishing the health examination $(n=207)$, those not completing the questionnaires $(n=233)$, and those who did not give consent to the study $(n=231)$. After these individuals were excluded, a total of 3924 subjects remained for subsequent analysis.

\section{Data collection \\ Anthropometry}

All demographic data were collected and recorded by trained staff. Weight and height were measured without shoes. Body mass index (BMI) was calculated as weight in kilograms divided by the height squared in meters $\left(\mathrm{kg} / \mathrm{m}^{2}\right)$.

\section{Covariates}

Participants completed a self-reported questionnaire (for details, see Additional file 1) on demographic factors, lifestyle factors, and dietary habits. Demographic factors included age, sex, residency, and education level. Lifestyle factors included physical activity, alcohol consumption, and smoking status. Dietary factors included milk consumption and taking calcium or vitamin D supplements.

\section{Assessment criteria}

We used the following five assessment criteria.

1) Physical exercise was defined as running, walking, dancing, tai chi, and ball games. Housework was not considered a form of physical exercise. High exercise levels were defined as more than $30 \mathrm{~min} /$ day or an average of more than $210 \mathrm{~min} /$ week. Low exercise levels were defined as less than $30 \mathrm{~min} /$ day or an average of less than $210 \mathrm{~min} /$ week. No exercise was considered as not performing any of the defined exercises for over 1 year.

2) Current smoker was defined as more than four cigarettes/week within 30 days prior to the health 
survey. Ex-smoker was defined as previous smoker, but with a current status of no smoking for at least 30 days. Subjects were classified as never smoking if they had no smoking history or if they smoked less than four cigarettes/week within 30 days prior to the health survey.

3) Consumption of $\geq 3$ alcoholic beverages/week was defined as more than 3 times/week, or an average daily amount of alcohol consumption more than 3 units/week for at least 5 years without interruption [per the standardized alcohol consumption index in the Fracture Risk Assessment Tool (FRAX)]. Consumption of fewer than 3 alcoholic beverages/ week was defined as less than 3 times/week, or an average daily amount of alcohol consumption less than 3 units/week. No consumption of alcoholic drinks was defined as no history of alcohol.

4) Milk consumption was assessed based on the following three levels: $\geq 250 \mathrm{~mL} /$ day; $<250 \mathrm{~mL} /$ day but $\geq 50 \mathrm{~mL} /$ day; and $<50 \mathrm{~mL} /$ day (no milk consumption).

5) Taking vitamin $D$ or calcium supplements was defined as follows: $2800 \mathrm{IU}$ vitamin D/week at least for 1 year; and $>400 \mathrm{mg}$ calcium/day at least for 1 year.

\section{Measurement of serum 25(OH)D levels}

Blood samples were obtained from study subjects in June-July 2014. Serum 25(OH)D levels were measured using standard method. Participants were instructed to fast for at least $12 \mathrm{~h}$ before analysis. Fasting blood samples were collected in the morning, immediately centrifuged, and the supernatant was transferred to new tubes. The serum samples were sent to Shanghai Key Laboratory of Clinical Geriatric Medicine for analysis within $6 \mathrm{~h}$ of blood draw. Serum 25(OH)D levels were measured by performing competitive electrochemiluminescence immunoassays using the Elecsys 2010 system (Roche Diagnostics, US). The intra-assay coefficient of variation $(\mathrm{CV})$ was $2.8 \%$, and the inter-assay $\mathrm{CV}$ was approximately $3.0-3.6 \%$. The lower limit of detection was $4 \mathrm{ng} / \mathrm{mL}$.

\section{Statistical analysis}

Statistical analysis was performed by SAS version 19.2. Categorical variables were considered as amounts and percents; continuous variables were considered as median and percentile. Descriptive statistics (frequencies, median, and quartile) were used to evaluate serum 25(OH)D levels. Data normality was assessed by Kolmogorov-Smirnov test. Serum 25(OH)D levels and impact factors were not normally distributed; thus, they were analyzed using a non-parametric test. Differences in gender characteristics and between subjects with serum $25(\mathrm{OH}) \mathrm{D}$ levels $\geq 20 \mathrm{ng} / \mathrm{mL}$ or $<20 \mathrm{ng} / \mathrm{mL}$ were compared using an independent $t$-test for normally distributed data or a Wilcoxon rank sum test for skewed data. Logistic regression models were developed to analyze associations between vitamin $\mathrm{D}$ deficiency and possible risk factors. Adjusted odds ratios (ORs) were calculated using serum $25(\mathrm{OH}) \mathrm{D}<20 \mathrm{ng} / \mathrm{ml}$ as the dependent variable and serum $25(\mathrm{OH}) \mathrm{D} \geq 20 \mathrm{ng} / \mathrm{mL}$ as the reference category. Univariate analysis was performed to arrive at a final model that included significant $(p<0.2)$ independent variables in the adjusted logistic regression analysis. Differences were considered as statistically significant at $p<0.05$.

\section{Results}

\section{Characteristics of the study population}

The general characteristics of the study subjects were presented in Table 1. A total of 3924 participants (1677 men and 2247 women) were included in this study. The mean age was 72 years for both genders. Factors significantly differed between male and female, including education level, physical activity, alcohol consumption, cigarette smoking, milk consumption, and supplementation with calcium or vitamin D. By contrast, demographic factors did not significantly differ including age, BMI and residency between men and women. These results suggest that gender-specific differences in vitamin $\mathrm{D}$ levels may not be due to demographic factors.

\section{Vitamin D levels in study subjects and the prevalence of vitamin $D$ deficiency in older adults}

Serum 25(OH)D levels were relatively skewed for study subjects of both genders; the median and mean vitamin D levels for men were 22.73 and $24.1 \mathrm{ng} / \mathrm{mL}$, respectively, and for women were 19.99 and $21.0 \mathrm{ng} / \mathrm{mL}$, respectively. Approximately 1693 (43.1\%) subjects had vitamin $\mathrm{D}$ deficiency [25(OH)D concentration $<20 \mathrm{ng} /$ $\mathrm{mL}$ ], and 1505 (38.4\%) had vitamin D insufficiency [25(OH)D concentration of 20-29.9 ng/mL]. The prevalence of vitamin D deficiency and insufficiency in men was 35.5 and $42.0 \%$, respectively; that in women was 48.9 and $35.7 \%$, respectively. Our data show that $4.6 \%$ of subjects $(2.73 \%$ of men and $5.9 \%$ of women) had severe vitamin D deficiency $[25(\mathrm{OH}) \mathrm{D}$ concentration $<10 \mathrm{ng} / \mathrm{mL}$ ]. Only $17.5 \%$ of subjects $(22.77 \%$ of men and $13.76 \%$ of women) had optimal vitamin D levels $[25(\mathrm{OH}) \mathrm{D}$ concentration $\geq 30 \mathrm{ng} / \mathrm{mL})$.

\section{Relationships between socioeconomic/sociodemographic} factors and vitamin D levels in older adults

The relationships between socioeconomic and sociodemographic factors and serum $25(\mathrm{OH}) \mathrm{D}$ levels in older adults are presented in Table 2. Significant differences were observed in serum $25(\mathrm{OH}) \mathrm{D}$ levels with respect to age, sex, education level, physical activity, alcohol consumption, cigarette smoking, supplementation with 
Table 1 Descriptive characteristics of the study population ${ }^{\text {a) }}$

\begin{tabular}{|c|c|c|c|c|c|c|}
\hline & & \multicolumn{2}{|l|}{ Male } & \multicolumn{2}{|l|}{ Female } & \multirow[t]{2}{*}{$P$ value } \\
\hline & & Median & $P_{25}-P_{75}$ & Median & $P_{25}-P_{75}$ & \\
\hline BMl & & 23.67 & $21.72-25.91$ & 23.82 & $21.77-26.06$ & 0.1450 \\
\hline \multirow[t]{2}{*}{ age } & & 72.00 & $68.00-78.00$ & 72.00 & $68.00-78.00$ & 0.3835 \\
\hline & & $N(\%)$ & & $N(\%)$ & & \\
\hline \multirow[t]{4}{*}{ Education } & Primary education & $337(20.10)$ & & $661(29.42)$ & & $<0.0001$ \\
\hline & Middle education & $749(44.66)$ & & $896(39.88)$ & & \\
\hline & College or above & $469(27.97)$ & & $283(12.59)$ & & \\
\hline & Under primary & $122(7.27)$ & & $407(18.11)$ & & \\
\hline \multirow[t]{3}{*}{ Physical exercise } & Never & $322(19.20)$ & & $541(24.08)$ & & 0.0003 \\
\hline & Low & $548(32.68)$ & & 744 (33.11) & & \\
\hline & High & $807(48.12)$ & & $962(42.81)$ & & \\
\hline \multirow[t]{3}{*}{ Alcohol consumption } & Never & $1092(65.12)$ & & $2179(96.97)$ & & $<0.0001$ \\
\hline & $<3$ drink /week & $340(20.27)$ & & $60(2.67)$ & & \\
\hline & $\geq 3$ drink /week & $245(14.61)$ & & $8(0.36)$ & & \\
\hline \multirow[t]{3}{*}{ Cigarette smoking } & Never & $1028(61.30)$ & & $2216(98.62)$ & & $<0.0001$ \\
\hline & Ex-smoker & $217(12.94)$ & & $2(0.09)$ & & \\
\hline & Current smoker & $432(25.76)$ & & $29(1.29)$ & & \\
\hline \multirow[t]{3}{*}{ Milk consumption } & Never & 665 (39.65) & & $672(29.91)$ & & $<0.0001$ \\
\hline & $<250 \mathrm{ml} /$ day & $696(41.50)$ & & $1099(48.91)$ & & \\
\hline & $\geq 250 \mathrm{ml} /$ day & $316(18.84)$ & & $476(21.18)$ & & \\
\hline \multirow[t]{2}{*}{ Calcium or vitamin D supplements } & No & $1461(87.12)$ & & $1770(78.77)$ & & $<0.0001$ \\
\hline & Yes & $216(12.88)$ & & $477(21.23)$ & & \\
\hline \multirow[t]{2}{*}{ Community } & urban & $1105(65.89)$ & & $1451(64.57)$ & & 0.3919 \\
\hline & suburb & $572(34.11)$ & & 796 (35.43) & & \\
\hline
\end{tabular}

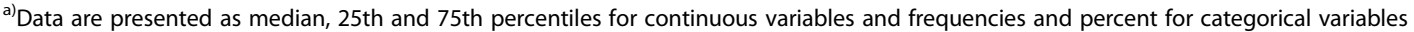

calcium or vitamin D, BMI, and residence in urban or suburban communities. We considered that confounding factors could modify these correlations. Therefore, single factors with significance of $P<0.2$ were selected for further multi-factor logistic regression analysis.

\section{Factors associated with vitamin D deficiency}

For logistic regression analyses of correlating factors, all subjects were assigned to one of two groups as follows: serum $25(\mathrm{OH}) \mathrm{D}<20 \mathrm{ng} / \mathrm{mL}$ or $25(\mathrm{OH}) \mathrm{D} \geq 20 \mathrm{ng} / \mathrm{mL}$. The regression analyses identified socioeconomic/sociodemographic factors associated with serum 25(OH)D deficiency (Tables 3 and 4). Sex, age, education level, physical exercise, alcohol consumption, smoking, milk consumption, supplementation with calcium or vitamin D, BMI, and residence in urban or suburban communities were included in the logistic regression analysis. Genderspecific lifestyle differences were used to build the statistic models. The results showed that education level and physical activity were significant correlating factors for men, whereas milk consumption and BMI were significant correlating factors for women (Tables 3 and 4). Taking calcium or vitamin D supplements and residence in urban or suburban communities were significant correlating factors for both genders (Tables 3 and 4).

For men (Table 3), low education level (uneducated or less than primary school) was a significant risk factor for serum 25(OH)D deficiency $(\mathrm{OR}>1)$, whereas physical exercise (duration $\geq 30 \mathrm{~min}$ ), calcium or vitamin $\mathrm{D}$ supplementation, and residence in suburban community were significant protective factors $(\mathrm{OR}<1)$. Vitamin $\mathrm{D}$ deficiency was more prevalent among male subjects with low education levels than in those who had at least completed primary school $(\mathrm{OR}=1.980,95 \%$ CI 1.266-3.098, $P=0.0115)$. However, there were no differences in vitamin D status among men with primary school, high school, or college degrees $(P>0.05)$. Physical exercise was a significant factor associated with vitamin $D$ status. Male subjects with $\geq 30 \mathrm{~min} /$ day physical exercise had significantly lower rates of vitamin $\mathrm{D}$ deficiency than those with no exercise (OR $=0.634,95 \%$ CI $0.479-0.839$, $P=0.0086)$; however, there was no significant difference in vitamin $\mathrm{D}$ status among subjects with no exercise and those who exercised for $<30 \mathrm{~min} /$ day. Male subjects who 
Table 2 Vitamin D status according to different characteristics of the study population

\begin{tabular}{|c|c|c|c|c|c|}
\hline & & \multicolumn{3}{|c|}{$\begin{array}{l}\text { Vitamin D } \\
\text { concentrations }\end{array}$} & \multirow[t]{2}{*}{$P$ value } \\
\hline & & Median & $P_{25}$ & $P_{75}$ & \\
\hline \multirow[t]{2}{*}{ Sex } & Male & 22.73 & 17.63 & 28.92 & \multirow[t]{2}{*}{$<0.0001$} \\
\hline & Female & 19.99 & 15.38 & 25.62 & \\
\hline \multirow[t]{4}{*}{ Age $^{a}$} & Q1 & 21.92 & 16.29 & 27.55 & \multirow[t]{4}{*}{0.0006} \\
\hline & Q2 & 21.18 & 16.39 & 27.26 & \\
\hline & Q3 & 21.07 & 16.44 & 27.15 & \\
\hline & Q4 & 20.52 & 15.24 & 26.76 & \\
\hline \multirow[t]{4}{*}{ Education level } & $\begin{array}{l}\text { Primary } \\
\text { education }\end{array}$ & 22.17 & 16.77 & 29.41 & \multirow[t]{4}{*}{$<0.0001$} \\
\hline & $\begin{array}{l}\text { Middle } \\
\text { education }\end{array}$ & 20.67 & 15.90 & 26.29 & \\
\hline & $\begin{array}{l}\text { College or } \\
\text { above }\end{array}$ & 21.04 & 16.16 & 26.35 & \\
\hline & Under primary & 20.40 & 15.92 & 26.47 & \\
\hline \multirow[t]{3}{*}{ Physical exercise } & Never & 20.01 & 14.95 & 27.15 & \multirow[t]{3}{*}{$<0.0001$} \\
\hline & Low & 20.76 & 16.04 & 26.25 & \\
\hline & High & 21.70 & 16.78 & 27.64 & \\
\hline \multirow[t]{3}{*}{ Alcohol consumption } & Never & 20.64 & 15.84 & 26.51 & \multirow[t]{3}{*}{$<0.0001$} \\
\hline & $<3$ drink/week & 22.76 & 17.54 & 29.74 & \\
\hline & $\geq 3 \mathrm{drink} /$ week & 24.23 & 18.86 & 31.23 & \\
\hline \multirow[t]{3}{*}{ Cigarette smoking } & Never & 20.66 & 15.93 & 26.55 & \multirow[t]{3}{*}{$<0.0001$} \\
\hline & Ex-smoker & 23.16 & 17.68 & 29.82 & \\
\hline & Current smoker & 22.67 & 17.06 & 29.49 & \\
\hline \multirow[t]{3}{*}{ Milk consumption } & No & 21.16 & 16.32 & 27.32 & \multirow[t]{3}{*}{0.1909} \\
\hline & $<250 \mathrm{ml} /$ day & 20.74 & 15.90 & 26.93 & \\
\hline & $\geq 250 \mathrm{ml} /$ day & 21.43 & 16.40 & 26.80 & \\
\hline \multirow{2}{*}{$\begin{array}{l}\text { Calcium or vitamin D } \\
\text { supplements }\end{array}$} & No & 20.59 & 15.74 & 26.33 & \multirow[t]{2}{*}{$<0.0001$} \\
\hline & Yes & 23.57 & 18.20 & 29.93 & \\
\hline \multirow[t]{4}{*}{$\mathrm{BMI}^{\mathrm{b}}$} & Q1 & 21.42 & 16.03 & 28.72 & \multirow[t]{4}{*}{0.0012} \\
\hline & Q2 & 21.59 & 16.60 & 27.25 & \\
\hline & Q3 & 20.83 & 16.18 & 26.75 & \\
\hline & Q4 & 20.45 & 15.68 & 25.69 & \\
\hline \multirow[t]{2}{*}{ Community } & urban & 20.16 & 15.44 & 25.65 & \multirow[t]{2}{*}{$<0.0001$} \\
\hline & suburb & 22.75 & 17.94 & 29.77 & \\
\hline
\end{tabular}

age: Q1: < 68 yr.; Q2: 68-71 yr.; Q3: $72-77$ yr.; Q4: $\geq 78$ yr

${ }_{B M I}\left(K g / m^{2}\right):$ Q1: $<21.76 ;$ Q2: 21.76-23.77; Q3: 23.77-25.99; Q4: $\geq 25.99$

took calcium or vitamin D supplements or lived in suburban communities had significantly lower prevalence of vitamin $\mathrm{D}$ deficiency than those who did not take supplements and lived in urban communities $(p<0.0001$ and $p=0.0012$, respectively).

For women (Table 4), milk consumption $(\geq 250 \mathrm{~mL} /$ day), taking calcium or vitamin D supplements, BMI, and residence in suburban communities were significant protective factors against vitamin $\mathrm{D}$ deficiency $(\mathrm{OR}<1$, $p<0.05$ ). By contrast, BMI $\geq 26.06 \mathrm{~kg} / \mathrm{m}^{2}$ was a significant risk factor for vitamin $\mathrm{D}$ deficiency $(\mathrm{OR}>1)$. Older female subjects with $\mathrm{BMI} \geq 26.06 \mathrm{~kg} / \mathrm{m}^{2}$ had higher vitamin $\mathrm{D}$ deficiency rates than those with $\mathrm{BMI}<21.77 \mathrm{~kg} /$ $\mathrm{m}^{2}(\mathrm{OR}=1.539,95 \%$ CI $1.199-1.974, P=0.0013)$. Older female subjects who consumed $\geq 250 \mathrm{~mL} /$ day of milk had a lower prevalence of vitamin D deficiency than those who did not drink milk (OR $=0.691,95 \%$ CI 0.532 $-0.898, P=0.0126$ ). Taking calcium or vitamin $\mathrm{D}$ supplements and living in suburban communities were strongly correlated with vitamin D sufficiency $(p<0.0001$ for both).

\section{Discussion}

We investigated vitamin D status with a large cohort of older adults in urban and suburban communities in Shanghai, and compared these data with an array of socioeconomic/sociodemographic factors. Our analyses indicate that vitamin D deficiency is highly prevalent among the older adult Chinese population. More than $82.5 \%$ of our subjects had serum $25(\mathrm{OH}) \mathrm{D}$ concentrations less than $30 \mathrm{ng} / \mathrm{mL}$, and approximately $43 \%$ had less than $20 \mathrm{ng} / \mathrm{mL}$, even during summer. Our linear regression analyses identified several potentially modifiable and gender-mediated factors that were independently associated with vitamin D deficiency. For men, low education level (below primary school) and low levels of physical activity (<30 min/day) were risk factors for vitamin D deficiency. For women, low milk consumption $(<250 \mathrm{~mL} /$ day $)$ and high BMI were risk factors for vitamin D deficiency. Residence in suburban communities and taking calcium or vitamin D supplements were protective factors for subjects of both sexes.

\section{Comparisons of vitamin D deficiency among older adults and other populations in China}

Our results on vitamin D deficiency of the older adults in Shanghai during summer were slightly lower than those reported previously for the same population and city during winter. The prevalence of vitamin $\mathrm{D}$ insufficiency $(<30 \mathrm{ng} / \mathrm{mL})$ and vitamin D deficiency $(<20 \mathrm{ng} /$ $\mathrm{mL}$ ) previously reported by Zhang et al. [9] was $84 \%$ and $30 \%$ in males, and $89 \%$ and $46 \%$ in females. In northeast China, vitamin D deficiency $(<25 \mathrm{nmol} / \mathrm{L})$ was observed in $48 \%$ and $15 \%$ of old men and women in early spring [10]. In central China, vitamin D sufficiency [serum $25(\mathrm{OH}) \mathrm{D} \geqq 75 \mathrm{nmol} / \mathrm{L}]$ was observed in $19.4 \%$ and $14.4 \%$ of men and women aged $60-89$ years, respectively [11]. A detailed multicenter study of healthy adults aged $>18$ years reported the following levels of vitamin D status in Chinese population: $5.9 \%$ had vitamin $\mathrm{D}$ severe deficiency $(<10 \mathrm{ng} / \mathrm{mL}$ ), 50.0\% had vitamin D deficiency (10-20 $\mathrm{ng} / \mathrm{mL}), 38.7 \%$ had vitamin D insufficiency (20 
Table 3 Logistic regression analysis for vitamin D deficiency by determinants in men

\begin{tabular}{|c|c|c|c|c|c|}
\hline & & $25(\mathrm{OH}) \mathrm{D} \mathrm{CC}$ & ion & $P$ value & OR $(95 \% \mathrm{Cl})$ \\
\hline & & $<20 \mathrm{ng} / \mathrm{L}$ & $\geq 20 \mathrm{ng} / \mathrm{L}$ & & \\
\hline & & $N(\%)$ & $N(\%)$ & & \\
\hline $\operatorname{Age}^{a}$ & Q1 & $108(6.55)$ & $211(12.79)$ & 0.5823 & 1.000 \\
\hline & Q2 & 173(10.48) & 298(18.06) & 0.5716 & $1.128(0.829-1.537)$ \\
\hline & Q3 & $143(8.67)$ & $278(16.85)$ & 0.3979 & $0.993(0.721-1.368)$ \\
\hline & Q4 & 171(10.36) & $268(16.24)$ & 0.2960 & $1.185(0.853-1.645)$ \\
\hline Education level & Primary education & $100(6.06)$ & 235(14.24) & 0.0059 & 1.000 \\
\hline & Middle education & $251(15.21)$ & 484(29.33) & 0.0564 & $1.157(0.850-1.575)$ \\
\hline & College or above & 188(11.39) & 272(16.48) & 0.3294 & $1.510(1.056-2.158)$ \\
\hline & Under primary & $56(3.39)$ & 64(3.88) & 0.0115 & $1.980(1.266-3.098)$ \\
\hline Physical exercise & Never & 138(8.36) & $172(10.42)$ & 0.0060 & 1.000 \\
\hline & Low & 197(11.94) & $347(21.03)$ & 0.3360 & $0.712(0.531-0.955)$ \\
\hline & High & $260(15.75)$ & $536(32.48)$ & 0.0086 & $0.634(0.479-0.839)$ \\
\hline Alcohol consumption & Never & $405(24.55)$ & $670(40.61)$ & 0.1102 & 1.000 \\
\hline & $<3$ drink/week & 118(7.15) & 217(13.15) & 0.9510 & $0.846(0.646-1.107)$ \\
\hline & $\geq 3$ drink/week & $72(4.36)$ & 168(10.18) & 0.1510 & $0.728(0.528-1.003)$ \\
\hline Cigarette smoking & Never & $366(22.18)$ & 646(39.15) & 0.3147 & 1.000 \\
\hline & Ex-smoker & $73(4.42)$ & $143(8.67)$ & 0.4040 & $0.958(0.692-1.326)$ \\
\hline & Current smoker & $156(9.45)$ & $266(16.12)$ & 0.1350 & $1.201(0.925-1.559)$ \\
\hline Milk consumption & No & $230(13.94)$ & $422(25.58)$ & 0.0998 & 1.000 \\
\hline & $<250 \mathrm{ml} /$ day & 265(16.06) & $429(26.00)$ & 0.0629 & $1.095(0.864-1.390)$ \\
\hline & $\geq 250 \mathrm{ml} /$ day & 100(6.06) & $204(12.36)$ & 0.0516 & $0.794(0.582-1.084)$ \\
\hline Calcium or vitamin D supplements & No & $542(32.85)$ & 893(54.12) & & 1.000 \\
\hline & Yes & $53(3.21)$ & 162(9.82) & $<0.0001$ & $0.488(0.347-0.686)$ \\
\hline $\mathrm{BMI}^{\mathrm{b}}$ & Q1 & 157(9.52) & 261(15.82) & 0.6327 & 1.000 \\
\hline & Q2 & 135(8.18) & $275(16.67)$ & 0.1989 & $0.834(0.621-1.122)$ \\
\hline & Q3 & $153(9.27)$ & 259(15.70) & 0.7678 & $0.966(0.719-1.298)$ \\
\hline & Q4 & 150(9.09) & $260(15.76)$ & 0.7410 & $0.970(0.719-1.307)$ \\
\hline Community & urban & $425(25.76)$ & 654(39.64) & & 1.000 \\
\hline & suburb & $170(10.30)$ & $401(25.87)$ & 0.0012 & $0.646(0.496-0.841)$ \\
\hline
\end{tabular}

$-30 \mathrm{ng} / \mathrm{mL}$ ), and $5.4 \%$ had vitamin $\mathrm{D}$ sufficiency (> $30 \mathrm{ng} / \mathrm{mL}$ ) [12].

Currently, there is a lack of standardization among studies investigating vitamin $\mathrm{D}$ levels with respect to the method of vitamin D measurement, and the definition of vitamin D deficiency and sufficiency, and seasonal variations. Other studies report that average serum $25(\mathrm{OH}) \mathrm{D}$ levels were lowest in winter and highest in summer $[13,14]$, and the prevalence rate of $25(\mathrm{OH}) \mathrm{D}$ deficiency was 4-5 times higher in winter-spring than that in summer-fall [15]. In our population, approximately $33 \%$ of men and $50 \%$ of women had deficient $25(\mathrm{OH}) \mathrm{D}$ concentrations $(<20 \mathrm{ng} / \mathrm{mL})$ in the summer, so we speculate that deficiency rates may be higher in other seasons. Consequently, it is likely that a large proportion of the older adults in Shanghai communities have suboptimal vitamin D concentrations, even during summer, when vitamin D levels should peak.

\section{Low milk consumption and high BMI were risk factors for vitamin $\mathrm{D}$ deficiency in women}

Food nutrients and nutritional supplements are known predictors of serum 25(OH)D levels [16, 17]. By comparison, few studies conducted in China have investigated the effects of dairy consumption or nutritional supplements on 25(OH)D levels. Our results indicate that milk consumption is more important in women than in men for vitamin D status, and milk consumption 
Table 4 Logistic regression analysis for vitamin D deficiency by determinants in women

\begin{tabular}{|c|c|c|c|c|c|}
\hline & & \multicolumn{2}{|c|}{ 25(OH)D concentration } & \multirow[t]{3}{*}{$P$ value } & \multirow[t]{3}{*}{ OR $(95 \% \mathrm{Cl})$} \\
\hline & & $<20 \mathrm{ng} / \mathrm{L}$ & $\geq 20 \mathrm{ng} / \mathrm{L}$ & & \\
\hline & & $N(\%)$ & $N(\%)$ & & \\
\hline \multirow[t]{4}{*}{$\mathrm{Age}^{\mathrm{a}}$} & Q1 & 208(9.47) & 237(10.79) & 0.6197 & 1.000 \\
\hline & Q2 & $311(14.16)$ & $320(14.57)$ & 0.9569 & $1.106(0.859-1.425)$ \\
\hline & Q3 & 291(13.25) & 285(12.98) & 0.6194 & 1.154(0.888-1.499) \\
\hline & Q4 & 288(13.11) & $256(11.66)$ & 0.3855 & $1.192(0.907-1.567)$ \\
\hline \multirow[t]{4}{*}{ Education level } & Primary education & 285(12.98) & $362(16.48)$ & 0.2082 & 1.000 \\
\hline & Middle education & $477(21.72)$ & 393(17.90) & 0.2841 & $1.237(0.975-1.568)$ \\
\hline & College or above & $142(6.47)$ & 133(6.06) & 0.7122 & $1.097(0.794-1.515)$ \\
\hline & Under primary & 194(8.83) & $210(9.56)$ & 0.3314 & $1.252(0.965-1.625)$ \\
\hline \multirow[t]{3}{*}{ Physical exercise } & Never & $270(25.96)$ & 243(11.07) & 0.4292 & 1.000 \\
\hline & Low & $372(16.94)$ & $364(16.58)$ & 0.5202 & $0.875(0.689-1.111)$ \\
\hline & High & $456(20.77)$ & $491(22.36)$ & 0.4029 & $0.866(0.689-1.089)$ \\
\hline \multirow[t]{3}{*}{ Milk consumption } & No & $341(15.53)$ & $321(14.16)$ & 0.0215 & 1.000 \\
\hline & $<250 \mathrm{ml} /$ day & $542(24.68)$ & $538(24.50)$ & 0.8547 & $0.845(0.687-1.040)$ \\
\hline & $\geq 250 \mathrm{ml} /$ day & $215(9.80)$ & 239(10.88) & 0.0126 & $0.691(0.532-0.898)$ \\
\hline \multirow[t]{2}{*}{ Calcium or vitamin D supplements } & No & $919(41.85)$ & 812(36.98) & & 1.000 \\
\hline & Yes & $179(8.15)$ & 286(13.02) & $<0.0001$ & $0.443(0.355-0.554)$ \\
\hline \multirow[t]{4}{*}{$\mathrm{BMI}{ }^{\mathrm{b}}$} & Q1 & $251(11.43)$ & $303(13.80)$ & 0.0068 & 1.000 \\
\hline & Q2 & $268(12.20)$ & $280(12.75)$ & 0.8450 & $1.178(0.922-1.506)$ \\
\hline & Q3 & $271(12.34 \%)$ & $278(12.66 \%)$ & 0.4542 & $1.129(0.882-1.445)$ \\
\hline & Q4 & $308(14.02 \%)$ & $237(10.79 \%)$ & 0.0013 & $1.539(1.199-1.974)$ \\
\hline \multirow[t]{2}{*}{ Community } & urban & 792(36.07) & $610(27.78)$ & & 1.000 \\
\hline & suburb & 306(13.93) & $488(22.22 \%)$ & $<0.0001$ & $0.394(0.314-0.493)$ \\
\hline
\end{tabular}

${ }^{\mathrm{a}}$ Age: Q1: < 68 yr.; Q2: 68-71 yr.; Q3: 72-77 yr.; Q4: $\geq 78 \mathrm{yr}$

${ }^{\mathrm{b}}$ BMI $\left(\mathrm{Kg} / \mathrm{m}^{2}\right)$ : Q1:<21.77; Q2: 21.77-23.81; Q3: 23.82-26.05; Q4: $\geq 26.06$

has a significant protective action against vitamin $\mathrm{D}$ deficiency in women when $>250 \mathrm{~mL}$ per day is consumed. A recent study among 504 older Singaporean Chinese adults also reported that $25(\mathrm{OH}) \mathrm{D}$ levels were associated with the vitamin $\mathrm{D}$, calcium, and dairy product intake for women but not for men [18]. Sex-mediated differences in dairy consumption and vitamin $\mathrm{D}$ status need to be clarified in future investigations.

Our study identified an inverse correlation between serum 25(OH)D levels and higher BMI for women but not for men. Older women with BMI $\geq 26.06 \mathrm{~kg} / \mathrm{m}^{2}$ had 1.539 times greater vitamin D deficiency rates than those with BMI $<21.77 \mathrm{~kg} / \mathrm{m}^{2}$. These results were consistent with those of a previous study [19], which also showed that BMI was independent predictors of blood $25(\mathrm{OH}) \mathrm{D}$ levels. Other studies in U.S. in immigrant populations reported that BMI is positively correlated with vitamin $\mathrm{D}$ deficiency, with a prevalence rate of $62.5 \%$ when BMI $\geq 25$ vs $56.2 \%$ when BMI $<25(P=0.02)$; however, this research included both men and women but did not differentiate effects of BMI by sex [20]. However, women have more body fat per unit weight than men [21], whereas vitamin D can be stored in fat, which may affect serum 25(OH)D levels. Other mechanism which can affect serum vitamin D levels should be lacking of outdoor activity in obese individuals $[22,23]$.

\section{Lack of physical exercise and low education level were risk factors of vitamin $D$ deficiency in men}

Our results indicate that $>30 \mathrm{~min} /$ day of physical exercise confers a significant protective effect against vitamin $\mathrm{D}$ deficiency in men, but not in women. A lack of regular physical activity (<30 min/day) was associated with vitamin D deficiency. Sociodemographic factors may influence this correlation. In China, older women traditionally perform all housework, and they enjoy evening activities such as walking and dancing; however, older men enjoy performing activities such as tai chi and jogging in the morning. Abboud et al. [24] reported that specific uptake of $25(\mathrm{OH}) \mathrm{D}$ into muscle may contribute to the long half-life of $25(\mathrm{OH}) \mathrm{D}$ in blood, and exercise may extend the half-life of serum $25(\mathrm{OH}) \mathrm{D}$. A recent 
study [25] also showed that physical activity was a significant positive determinant $(\beta=0.13)$ of serum vitamin D levels and smoking was found to be a significant negative determinant $(\beta=-2.64)$, the latter of which stands in contrast to the present study, which found no such correlation.

Our regression analyses also indicated that a lower education level (those who did not complete primary school) was a significant risk factor for vitamin D deficiency in older men. This result is consistent with that of a previous study, which showed that vitamin D deficiency was common among men with no education and a sedentary lifestyle [26]; however, another study from Southern China found that highly educated single females tend to be vitamin $\mathrm{D}$ deficient, which may be affected by a desire to be pale and sun avoidance [27]. We evaluated whether this correlation might be due to confounding factors (for example, men with low socioeconomic status may be prone to alcohol consumption and smoking); however, our analyses did not find any associations with potential confounding factors. Future studies will be required to clarify the observed correlation between low education and vitamin $\mathrm{D}$ deficiency among older men.

\section{Calcium or vitamin $\mathrm{D}$ supplementation and residence in suburban communities were predictors of vitamin $D$ sufficiency for older men and women}

We identified an association between serum 25(OH)D concentration and resident location (urban or suburban). To our knowledge, this has not been investigated in previous studies conducted in China. We found that older residents in suburban communities had a lower prevalence of vitamin D deficiency than those in urban communities. This may be due to lifestyle differences, such as the amount of outdoor activity (higher in suburban communities) and the use of sun protection tools (higher in urban communities).

Regarding calcium or vitamin D supplements, our results indicate that subjects who take calcium or vitamin D supplements have significantly higher $25(\mathrm{OH}) \mathrm{D}$ levels than those who do not. Chan et al. [28] also reported that serum $25(\mathrm{OH}) \mathrm{D}$ concentration was associated with vitamin $\mathrm{D}$ supplementation rather than dietary habit. A study from Brolsma EM et al. [29] investigated determinants of vitamin deficiency in an older population, and found that sun exposure, genetic background and vitamin D intake were main factors explaining $35 \%$ of $25(\mathrm{OH}) \mathrm{D}$ status. Brolsma et al. also asserted that vitamin D supplement may be an inexpensive, easy and effective strategy to prevent vitamin D deficiency.

Vitamin D supplements can enhance vitamin D levels, and calcium supplements may facilitate vitamin D utilization. Low levels of dietary calcium intake may give rise to mild hyperparathyroidism and accelerate the conversion of $25(\mathrm{OH}) \mathrm{D}$ into $1,25(\mathrm{OH}) 2 \mathrm{D}$, which consequently reduces $25(\mathrm{OH}) \mathrm{D}$ levels [30]. Our results indicate that low vitamin D supplement intake $(<400 \mathrm{IU} /$ day) did not significantly affect vitamin $\mathrm{D}$ status; however, intake of $\geq 400 \mathrm{IU} /$ day vitamin $\mathrm{D}$ supplement maintained serum 25(OH)D concentration $>20 \mathrm{ng} / \mathrm{mL}$. Few foods naturally contain vitamin $\mathrm{D}$, and no foods are fortified with vitamin D in China; therefore, older adults in China must depend on taking nutritional supplements to maintain vitamin D sufficiency. In our study, taking either calcium or vitamin D supplements had a protective effect against vitamin D deficiency, provided the average calcium or vitamin D intake was $>400 \mathrm{mg}$ or $>400$ IU per day, respectively.

\section{Sub-group analyses \\ Age}

There were no significant associations between age and serum 25(OH)D concentrations in our study cohort (aged $>65$ years). Previous studies reported decreased cutaneous production of vitamin D with age [31,32]. Our study did not observe significant differences in serum 25(OH)D levels in people 65-95 years old for either sex, suggesting that age is not a correlating factor within this population. Further studies are required to explore possible stratifying effects of age among older adults.

\section{Gender}

Our results showed that old women tended to have lower serum $25(\mathrm{OH}) \mathrm{D}$ levels than those of old men, which is consistent with other studies [33, 34]. Data from these studies indicate that, compared to women, men had higher $25(\mathrm{OH}) \mathrm{D}$ levels regardless of BMI, which is apparently due to the greater body surface area of men that can absorb sunlight. Furthermore, men work outdoors more often.

\section{Conclusions}

Vitamin D deficiency is high prevalent in older adults in China, and many modifiable lifestyle factors are potentially important determinant on vitamin D deficiency. This study showed that taking calcium or vitamin D supplements and living in suburban communities were predictors of vitamin D sufficiency for both sexes. For women, low levels of milk consumption and high BMI were significantly correlated with vitamin D deficiency. For men, low education levels and low physical activity were significantly correlated with vitamin D deficiency. Further studies are wanted on potential correlation mechanisms and optimal strategies to improve vitamin D status in community old population. The results indicated that community old adults should be encouraged to consume more foods with vitamin $\mathrm{D}$, take calcium 
and vitamin D supplements, drink more milk, and participate in physical exercise to improve their health.

\section{Additional files}

Additional file 1: Bone health questionnaire. (DOCX $18 \mathrm{~kb}$ )

\section{Abbreviations}

25(OH)D: 25-hydroxyvitamin D; BMI: Body mass index; CV: Coefficient of variation; ECL: Electrochemiluminescence; FRAX: Fracture Risk Assessment Tool; LC/MS-MS: Liquid chromatography-tandem mass spectrometry; OR: Odds ratios; PTH: Parathyroid hormone; SERMs: Selective estrogen receptor modulators

\section{Acknowledgements}

We like to thank a bunch to Yang Fei about her work on statistical analysis of data in this study, and we also like to thank all participants of the study and the involved laboratory staff.

We also give great thank to BioMed Proofreading ${ }^{\circledR}$ LLC from which this manuscript has been edited by native English-speaking experts. We thank all participants of the study and the laboratory staff who helped conduct the study.

This study was supported by the National Natural Science Foundation of China (NSFC; No. 81471089), Shanghai Municipal Commission of Health (2013ZYJB0801), and Shanghai Key Laboratory of Clinical Geriatric Medicine $(13 \mathrm{dz} 2260700)$

\section{Funding}

This study was supported by the National Natural Science Foundation of China (NSFC) (No. 81471089), Shanghai Municipal Commission of Health (2013ZYJB0801) and Shanghai Key Laboratory of Clinical Geriatric Medicine $(13 \mathrm{dz} 2260700)$

\section{Availability of data and materials}

Our data will not be shared because of the grant support this research hasn't been done yet, and all the data was confidentiality.

\section{Authors' contributions}

QC involved in the design of the study and interpretation of data; YPD and WH participated in acquisition of data, analysis and interpretation of data; WJT and HLL participated in the study implementation and coordination; MMC involved in drafting the manuscript; SBZ gave final approval of the version to be published. All authors read and approved the final manuscript.

\section{Ethics approval and consent to participate}

All of the subjects provided written informed consent before participating in the study, and the program was approved by the Huadong Hospital Ethics Committee (Project NO.2014 K004).

\section{Consent for publication}

Not Applicable.

\section{Competing interests}

The authors declare that they have no competing interests.

\section{Publisher's Note}

Springer Nature remains neutral with regard to jurisdictional claims in published maps and institutional affiliations.

\section{Author details}

${ }^{1}$ Department of Osteoporosis and Bone Disease, Research Section of Geriatric Metabolic Bone Disease, Shanghai Geriatric Institute, Huadong Hospital affiliated to Fudan University, Shanghai, China. ${ }^{2}$ Research Center on Aging and Medicine, Fudan University, 221 West Yan An Road, Shanghai 200040, China.
Received: 4 January 2017 Accepted: 8 October 2017

Published online: 24 October 2017

\section{References}

1. Mithal A, Wahl DA, Bonjour JP, Burckhardt P, Dawson-Hughes B, Eisman JA El-Hajj Fuleihan G, Josse RG, Lips P, Morales-Torres J, IOF Committee of Scientific Advisors (CSA) Nutrition Working Group. Global vitamin D status and determinants of hypovitaminosis D. Osteoporos Int. 2009;20(11):1807-20.

2. Brincat $M$, Gambin J, Brincat $M$, Calleja-Agius J. The role of vitamin D in osteoporosis. Maturitas. 2015;80(3):329-32.

3. Beveridge LA, Witham MD. Vitamin D and the cardiovascular system. Osteoporos Int. 2013;24(8):2167-80.

4. Zhang MX, Pan GT, Guo JF, Li BY, Qin LQ, Zhang ZL. Vitamin D Deficiency increases the risk of gestational diabetes mellitus: a meta-analysis of observational studies. Nutrients. 2015;7(10):8366-75.

5. Khayatzadeh S, Feizi A, Saneei P, Esmaillzadeh A. Vitamin D intake, serum vitamin D levels, and risk of gastric cancer: a systematic review and metaanalysis. J Res Med Sci. 2015;20(8):790-6.

6. Rolf $L$, Muris AH, Hupperts R, Damoiseaux J. Illuminating vitamin D effects on B-cells - the multiple sclerosis perspective. Immunology. 2016;147(3): 275-84.

7. Arabi A, El Rassi R, El-Hajj Fuleihan G. Hypovitaminosis D in developing countries-prevalence, risk factors and outcomes. Nat Rev Endocrinol. 2010; 6(10):550-61.

8. Cheng Q, Zhu YX, Zhang MX, Li LH, PY D, Zhu MH. Age and sex effects on the association between body composition and bone mineral density in healthy Chinese men and women. Menopause. 2012;19(4):448-55.

9. HK L, Zhang Z, Ke YH, He JW, WZ F, Zhang CQ, Zhang ZL. High prevalence of vitamin D insufficiency in China: relationship with the levels of parathyroid hormone and markers of bone turnover. PLoS One. 2012;7(11): e47264.

10. Yan L, Prentice A, Zhang H, Wang X, Stirling DM, Golden MM. Vitamin D status and parathyroid hormone concentrations in Chinese women and men from north-east of the People's Republic of China. Eur J Clin Nutr. 2000:54(1):68-72.

11. Feng X, Guo T, Wang Y, Kang D, Che X, Zhang H, Cao W, Wang P. The vitamin $D$ status and its effects on life quality among the elderly in Jinan, China. Arch Gerontol Geriatr. 2016;62:26-9.

12. Yu S, Fang H, Han J, Cheng X, Xia L, Li S, Liu M, Tao Z, Wang L, Hou L, Oin $X$, Li P, Zhang R, Su W, Qiu L. The high prevalence of hypovitaminosis D in China: a multicenter vitamin D status survey. Medicine (Baltimore). 2015; 94(8):e585.

13. Kull M Jr, Kallikorm R, Tamm A, Lember M. Seasonal variance of 25-(OH) vitamin $D$ in the general population of Estonia, a northern European country. BMC Public Health. 2009;9:22

14. Merlo C, Trummler M, Essig S, Zeller A, Vitamin D. Deficiency in unselected patients from Swiss primary care: a cross-sectional study in TwoSeasons, e0138613. PLoS One. 2015:10(9)

15. Choi EY. 25(OH)D status and demographic and lifestyle determinants of 25(OH)D among Korean adults. Asia Pac J Clin Nutr. 2012;21:526-35.

16. Al-Daghri NM, Aljohani N, Al-Attas OS, Krishnaswamy S, Alfawaz H, Al-Ajlan A, Alokail MS. Dairy products consumption and serum 25-hydroxyvitamin D level in Saudi children and adults. Int J Clin Exp Pathol. 2015;8(7):8480-6.

17. Kimlin MG, Lucas RM, Harrison SL, van der Mei I, Armstrong BK, Whiteman DC, Kricker A, Nowak M, Brodie AM, Sun J. The contributions of solar ultraviolet radiation exposure and other determinants to serum 25hydroxyvitamin D concentrations in Australian adults: the AusD study. Am J Epidemiol. 2014;179(7):864-74.

18. Robien K, Butler LM, Wang R, Beckman KB, Walek D, Koh WP, Yuan JM. Genetic and environmental predictors of serum 25-hydroxyvitamin D concentrations among middle-aged and elderly Chinese in Singapore. $\mathrm{Br}$ JNutr. 2013;109:493-502.

19. Ardawi MS, Qari MH, Rouzi AA, Maimani AA, Raddadi RM. Vitamin D status in relation to obesity, bone mineral density, bone turnover markers and vitamin D receptor genotypes in healthy Saudi pre- and postmenopausal women. Osteoporos Int. 2011;22(2):463-75.

20. Campagna AM, Settgast AM, Walker PF, DeFor TA, Campagna EJ, Plotnikoff GA. Effect of country of origin, age, and body mass index on prevalence of vitamin D deficiency in a US immigrant and refugee population. Mayo Clin Proc. 2013;88(1):31-7. 
21. Cheng Q, Zhu X, Zhang X, Li H, Du Y, Hong W, Xue S, Zhu H. A crosssectional study of loss of muscle mass corresponding to sarcopenia in healthy Chinese men and women: reference values, prevalence, and association with bone mass. J Bone Miner Metab. 2014:32(1):78-88.

22. McCullough ML, Weinstein SJ, Freedman DM, Helzlsouer K, Flanders WD, Koenig K, Kolonel L, Laden F, Le Marchand L, Purdue M, et al. Correlates of circulating 25-hydroxyvitamin D: cohort consortium vitamin D pooling project of rarer cancers. Am J Epidemiol. 2010;172:21-35.

23. Larose TL, Chen Y, Camargo CA Jr, Langhammer A, Romundstad P, Mai XM. Factors associated with vitamin D deficiency in a Norwegian population: the HUNT study. J Epidemiol Community Health. 2014;68:165-70.

24. Abboud M, Puglisi DA, Davies BN, Rybchyn M, Whitehead NP, Brock KE, Cole L, Gordon-Thomson C, Fraser DR, Mason RS. Evidence for a specific uptake and retention mechanism for 25-hydroxyvitamin D (25OHD) in skeletal muscle cells. Endocrinology. 2013:154(9):3022-30.

25. Cheng TY, Millen AE, Wactawski-Wende J, Beresford SA, LaCroix AZ, Zheng Y, Goodman GE, Thornquist MD, Neuhouser ML. Vitamin D intake determines vitamin d status of postmenopausal women, particularly those with limited sun exposure. J Nutr. 2014;144(5):681-9.

26. Ardawi MS, Sibiany AM, Bakhsh TM, Qari MH, Maimani AA. High prevalence of vitamin $D$ deficiency among healthy Saudi Arabian men: relationship to bone mineral density, parathyroid hormone, bone turnover markers, and lifestyle factors. Osteoporos Int. 2012;23(2):675-86.

27. Ke L, Mason RS, Mpofu E, Dibley M, Li Y, Brock KE. Vitamin D and parathyroid hormone status in a representative population living in Macau, China. J Steroid Biochem Mol Biol. 2015;148:261-8.

28. Chan J, Jaceldo-Siegl K, Fraser GE. Serum 25-hydroxyvitamin D status of vegetarians, partial vegetarians, and nonvegetarians: the Adventist health Study-2. Am J Clin Nutr. 2009;89(5):1686S-92S.

29. EM B-B, AM V, NL $\vee d Z$ Z, JP $\vee W, K M S, A C H, S C \vee D, A W E, E S, N M \vee S, N \vee d V$, $A G U, P L, E J F, R A D-R$, de Groot LC. Relative importance of summer sun exposure, vitamin D intake, and genes to vitamin D status in Dutch older adults: the B-PROOF study. J Steroid Biochem Mol Biol. 2016;164:168-76.

30. Lips P. Interaction between vitamin D and calcium. Scand J Clin Lab Invest Suppl. 2012;243:60-4.

31. Holick MF, Matsuoka LY, Wortsman J. Age, vitamin D, and solar ultraviolet. Lancet. 1989;2:1104-5.

32. Need AG, Morris HA, Horowitz M, et al. Effects of skin thickness, age, body fat, and sunlight on serum 25-hydroxyvitamin D. Am J Clin Nutr. 1993;58: 882-5.

33. Pazaitou-Panayiotou K, Papapetrou PD, Chrisoulidou A, Konstantinidou S, Doumala E, Georgiou E, Panagiotou V, Sotiriadou E, Mavroudi E, ApostolakiChristopoulou M. Height, whole body surface area, gender, working outdoors, and sunbathing in previous summer are important determinants of serum 25-hydroxyvitamin D levels. Exp Clin Endocrinol Diabetes. 2012; 120(1):14-22.

34. Van Dam RM, Snijder MB, Dekker JM, Stehouwer CD, Bouter LM, Heine RJ, Lips P. Potentially modifiable determinants of vitamin D status in an older population in the Netherlands: the Hoorn study. Am J Clin Nutr. 2007;85: 755-61.

\section{Submit your next manuscript to BioMed Central and we will help you at every step:}

- We accept pre-submission inquiries

- Our selector tool helps you to find the most relevant journal

- We provide round the clock customer support

- Convenient online submission

- Thorough peer review

- Inclusion in PubMed and all major indexing services

- Maximum visibility for your research

Submit your manuscript at www.biomedcentral.com/submit

Biomed Central 